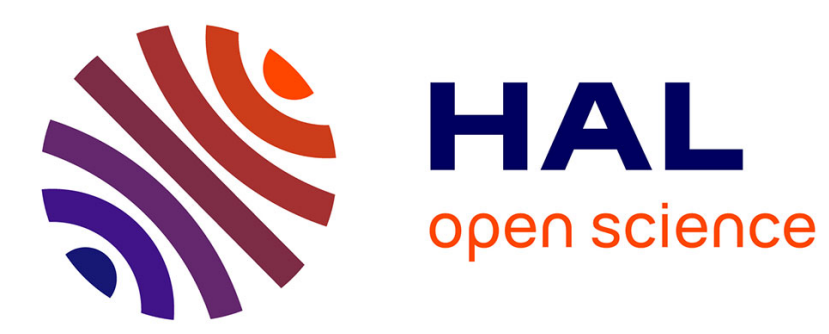

\title{
Inverse transition of labyrinthine domain patterns in ferroelectric thin films
}

Y. Nahas, S. Prokhorenko, J. Fischer, B. Xu, C. Carretero, S. Prosandeev, M. Bibes, S. Fusil, B. Dkhil, V. Garcia, et al.

\section{- To cite this version:}

Y. Nahas, S. Prokhorenko, J. Fischer, B. Xu, C. Carretero, et al.. Inverse transition of labyrinthine domain patterns in ferroelectric thin films. Nature, 2020, 10.1038/s41586-019-1845-4 . hal-02483177

\section{HAL Id: hal-02483177 \\ https://hal.science/hal-02483177}

Submitted on 18 Feb 2020

HAL is a multi-disciplinary open access archive for the deposit and dissemination of scientific research documents, whether they are published or not. The documents may come from teaching and research institutions in France or abroad, or from public or private research centers.
L'archive ouverte pluridisciplinaire HAL, est destinée au dépôt et à la diffusion de documents scientifiques de niveau recherche, publiés ou non, émanant des établissements d'enseignement et de recherche français ou étrangers, des laboratoires publics ou privés. 


\title{
Inverse transition of labyrinthine domain patterns in ferroelectric thin films
}

\author{
Y. Nahas ${ }^{1}$, S. Prokhorenko' ${ }^{1}$ J. Fischer ${ }^{2}$, B. Xu ${ }^{3}$, C. Carrétéro ${ }^{2}$, S. \\ Prosandeev $^{1,4}$, M. Bibes ${ }^{2}$, S. Fusil ${ }^{2,5}$, B. Dkhil ${ }^{6}$, V. Garcia ${ }^{2}$, and L. Bellaiche ${ }^{1}$ \\ ${ }^{1}$ Physics Department and Institute for Nanoscience and Engineering, \\ University of Arkansas, Fayetteville, AR 72701, USA \\ ${ }^{2}$ Unité Mixte de Physique, CNRS, Thales, Univ. Paris Sud, \\ Université Paris-Saclay, Palaiseau 91767, France \\ ${ }^{3}$ School of Physical Science and Technology, Soochow University, Suzhou 215006, China \\ 4 Institute of Physics and Physics Department of Southern Federal University, Rostov-na-Donu 344090, Russia \\ 5 Université d'Evry, Université Paris-Saclay, 91025 Evry, France \\ ${ }^{6}$ Laboratoire Structures, Propriétés et Modélisation des Solides, CentraleSupélec, \\ UMR CNRS 8580, Université Paris-Saclay, 91190 Gif-sur-Yvette, France
}

Phase separation is a cooperative process whose kinetics underpins the orderly morphogenesis of domain patterns on mesoscopic scales $^{1,2}$. Systems of highly degenerate frozen states may exhibit the rare and counter-intuitive inverse symmetry breaking phenomenon ${ }^{3}$. Already hypothesized a century ago ${ }^{4}$, inverse transitions have been found experimentally in very disparate materials, ranging from polymeric and colloidal compounds to high- $T_{c}$ superconductors, proteins, ultrathin magnetic films, liquid crystals and metallic alloys ${ }^{5,6}$, with the notable exception of ferroelectric oxides, despite the widespread theoretical and experimental work on the latter. Here we show that subsequent to a subcritical quench, the non-equilibrium self-assembly of ferroelectric domains in ultrathin films of $\mathbf{P b}\left(\mathbf{Z r}_{0.4} \mathbf{T i}_{0.6}\right) \mathbf{O}_{3}$ (PZT) results in a maze, or labyrinthine pattern, featuring meandering stripe domains. Furthermore, upon increasing temperature, this highly degenerate labyrinthine phase undergoes an inverse transition whereby it transforms into the lesssymmetric parallel stripe domain structure, before the onset of paraelectricity at higher temperatures. We find that this phase sequence is ascribed to enhanced entropic contribution of domain walls, and that domain straightening and coarsening is predominantly driven by the relaxation and diffusion of topological defects. The computational modeling and the experimental observation of the inverse dipolar transition in $\mathrm{BiFeO}_{3}$ (BFO) suggest the universality of the phenomenon in ferroelectric oxides. The multitude of self-patterned states and the various topological defects they embody may be put at use to leap beyond current domain and domain-wall-based ${ }^{7}$ technologies by enabling fundamentally new design principles and topologically enhanced functionalities within ferroelectric films.

To investigate polarization self-patterning, we use an ab-initio based effective Hamiltonian approach ${ }^{35}$ and ex- amine ultrathin films of $\mathrm{Pb}\left(\mathrm{Zr}_{0.4} \mathrm{Ti}_{0.6}\right) \mathrm{O}_{3}$ (see Methods section), for these widely used quasi-two dimensional ferroelectric systems are already known to exhibit various modulated phases depending on the interplay between strain and the amount of screening of surface charges $^{8,9,34-36,43}$.

It is worthwhile noting that two underlying nested symmetry-breaking processes are at play in these systems and involve two distinct dynamical length scales. While compressive strain introduces crystalline anisotropy and favors dipoles with orientation perpendicular to the filmplane $^{1,35}$ (cubic symmetry is reduced to a quasi- $\mathbb{Z}_{2}$ symmetry), the depolarizing field arising from incomplete screening of surface charges essentially imposes zero net polarization, and favors instead the formation of multiple mesoscopic domains as a result of the spontaneous breaking of the residual discrete symmetry. These domains of opposite polarization alternate along in-plane directions, and consist each of ferroelectrically ordered ensembles of dipoles. More precisely, while an individual dipole retains the freedom to flip between [001] and [001] out-ofplane directions, an individual domain, as an emergent mesoscopic degree of freedom, has the propensity to align along either [100] (horizontal) or [010] (vertical) in-plane tetragonal directions, due to the underlying square lattice geometry ${ }^{1}$. Naturally, the dynamics pertaining to the motion and relaxation of domains is slower than that of individual dipole fluctuations, and this very fact poses important questions as to what extent domain dynamics and their morphology will be kinetically constrained.

One manifestation of this kinetic constraint resides in the possibility of obtaining two distinct modulated phases at low temperatures depending on the rate of cooling. While the well-known parallel stripe domain pattern (Fig. 1a) emerges as the ground state upon adiabatically cooling (annealing) the system ${ }^{34,35}$, the labyrinthine domain polarization pattern (Fig. 1b) onsets upon abruptly cooling (sub-critical quenching) the system . The latter pattern consists of convoluted stripes and meandering domains and has a very close internal energy that is only $0.6 \%$ higher than that of the ground state. Interest- 
ingly, inquiring into the stability of the labyrinthine state at $T \rightarrow 0 \mathrm{~K}$, we find that the eigenvalues of the Hessian matrix of the Hamiltonian are closely distributed around zero, with $75 \%$ of them being negative, hence indicating that the labyrinthine state is weakly unsta$\mathrm{ble}^{14}$. Furthermore, we find that at $10 \mathrm{~K}$, the labyrinthine state has quasi-vanishing relaxation rate without evidence of a growing static cooperative length, similarly to a glass-like kinetically arrested state ${ }^{15,16}$. The slightly off-equilibrium labyrinthine structure only asymptotically departs from the state in which it initially vitrified, hence being effectively stationary at $T \rightarrow 0 \mathrm{~K}$ in the thermodynamic limit. The frozen labyrinthine state retains some of the properties of the high-temperature paraelectric state (similarly to the common local structure exhibited by glasses and their liquid phases), such as the overall absence of long-range orientational order at the mesoscale mirrored by its structure factor that has a ring-shaped spectral weight (inset of Fig.2b). However, the spectral weight distribution is deformed by the underlying fourfold square lattice anisotropy (four-peaked crown), thus signaling that the labyrinthine domain pattern is only weakly disjoint from the square symmetry of the lattice geometry. In fact, upon sub-critically quenching the system, we discern a local tendency of adjacent domains to order by adopting one of the two lower equilibrium states of the Hamiltonian (either horizontal [100] or vertical [010] periodicity of parallel stripes is associated with the twofold-degenerate ground-state). This local ordering process ensures a local minimization of the energy and can extend only up to a certain finite length-scale, beyond which collective mismatch and surface tension effects hinder further ordering ${ }^{17,18}$. In this regard, the low-temperature labyrinthine state can be apprehended as a mosaic pattern consisting in a spatial mixture of tiles with different realization of local order. This labyrinthine state inherently features frustration due to the unresolvable competition between local interactions and the long-ranged dipolar interaction ${ }^{1,16,19}$.

Upon heating the labyrinthine state, thermal activation effects come into play and the resulting kinetic unfreezing elicits the phenomenon of inverse transition, whereby a state with higher symmetry transforms into a lower symmetry one. This is shown in Figs. $2 b$ to g) where, upon raising the temperature, the more symmetric labyrinthine phase obtained by quenching the system from $650 \mathrm{~K}$ to $10 \mathrm{~K}$, experiences a lessening of its junctions resulting in a transient reordering and the occurrence of the less symmetric parallel stripe state at higher temperatures. This inverse transition onsets at $T_{i n v} \sim 200 \mathrm{~K}$, before transiting to the paraelectric state at around $T_{c} \sim 380 \mathrm{~K}$ (Extended Data Figs. 1 and 2). As temperature increases, the distribution of the spectral weight of the structure factor gradually yields two primary spots along the direction of the Brillouin zone perpendicular to the direction of the stripes in the real space, mirroring the acquired long-range orientational order. This inverse symmetry breaking can be quantified using the order parameter $O_{h v}=\left(n_{h}-n_{v}\right) / n$, where $n$ is the total number of first nearest neighbor pairs of dipoles having opposite sign of their z-component, and $n_{h}\left(n_{v}\right)$ is the number of horizontal (vertical) bonds among such dipoles ${ }^{1,20}$. The average of this quantity over one hundred labyrinthine realizations is shown in Fig. 2a and its evolution with temperature captures the sequential onset of three distinct phases: a low-temperature labyrinthine phase with no net orientation that bears the symmetry of the underlying lattice, a mid-temperature broken-symmetry phase with distinguishable orientation of domains that are all oriented as stripes along a common axis, and a high-temperature disordered paraelectric phase characterized by the dissolution of domains and domain walls.

As a general energetic feature of domain walls within modulated phases ${ }^{1}$, we find that the gain realized by short-range interactions is counterbalanced by the cost of dipolar interaction that plays an important, if not dominant, role (Extended Data Figs. 3 and 4). The excess length of domain walls within the labyrinthine state therefore yields an excess in the dipolar cost when compared to the parallel stripe domain structure. We find that this excess gradually reduces with increasing temperature as a result of the straightening of meandering stripes, and vanishes at $\mathrm{T}_{i n v}$ (Extended Data Fig. 5a).

We experimentally observed such an inverse transition phenomenon within $\mathrm{BiFeO}_{3}$ (BFO) thin films (Fig. 3a), in agreement with our first-principles computations (Fig. 3c). The 95-nm-thick $\mathrm{BiFeO}_{3}$ layer was grown by pulsed laser deposition on a (110)-oriented orthorhombic $\mathrm{DyScO}_{3}$ substrate at $933 \mathrm{~K}$ (Extended Data Figs. 9 and 10) and exhibited, after having been cooled down to room temperature, a labyrinthine domain structure (Fig. 3a, as-grown). We have then performed series of experiments in which the as-grown sample was first annealed for one hour at an elevated target temperature and then cooled down to room temperature with an effective cooling rate of $2 \mathrm{~K} / \mathrm{min}$. The ferroelectric domain landscape observed at room temperature after annealing at $773 \mathrm{~K}, 1023 \mathrm{~K}$ and $1073 \mathrm{~K}$ is shown in Fig. 3a. As it can be readily seen, for target temperatures up to $1023 \mathrm{~K}$ the labyrinthine morphology is retained, while following the $1073 \mathrm{~K}$ annealing, a profound modification to a perfect stripe domain pattern onsets. The increased ordering of the ferroelectric array was confirmed macroscopically by $\mathrm{X}$-ray diffraction (XRD) measurements (Extended Data Fig. 12) following the pioneering work of Streiffer et al. ${ }^{36}$. These experiments indicate an inverse transition $\left(T_{i n v}\right)$ in between $1023 \mathrm{~K}$ and $1073 \mathrm{~K}$, while no transition to the paraelectric state could be detected by XRD up to $1160 \mathrm{~K}$ (Extended Data Fig. 11). We also found by performing conducting AFM measurements that elementary point defects (Fig. 3b) are characterized by enhanced conduc- 
tion that can be up to fifty times larger than the conduction at straight segments of domain walls. Indeed, we find that the typical current level is of $0.2 \mathrm{pA}$ in domains, 0.5-1 pA at domain walls, $15 \mathrm{pA}$ at end-point defects and $50 \mathrm{pA}$ at three fold junctions. The inverse transition in $\mathrm{BiFeO}_{3}$ is also seen in our first-principles effective Hamiltonian simulations ${ }^{49-51}$ that yield $T_{i n v} \sim 1100 \mathrm{~K}$ and $T_{c} \sim 1300 \mathrm{~K}$ for $\mathrm{BiFeO}_{3}$ films (Fig. 3c). Interestingly, the AFD degrees of freedom in $\mathrm{BiFeO}_{3}$, albeit coupled to the ferroelectric order parameter ${ }^{24}$, do not hamper the onset of the inverse transition (Extended Data Figs. 6 and 7 ). These first-principles obtained numerical results, along with our experimental findings and other computations given in the Methods section, demonstrate that the inverse transition phenomenon is robust against intrinsic and extrinsic parameters such as boundary conditions, film thickness, as well as screening conditions and misfit strain. Naturally, varying conditions in the studied systems (PZT and BFO) yield different transition temperatures, as well as different types of domain walls (e.g., $180^{\circ}$ in $\mathrm{PZT}$ versus $109^{\circ}$ and $71^{\circ}$ domain walls in $\mathrm{BFO}$ ) and labyrinthine morphologies, with no incidence on the occurrence of the inverse transition.

Figure 4a provides the evolution with temperature of the free-energy like potential associated with the transverse component of dipoles at the domain walls. Each curve is obtained upon averaging over the distributions of one hundred labyrinthine realizations. Results are gathered at $10 \mathrm{~K}, 110 \mathrm{~K}$, and $210 \mathrm{~K}$ upon heating the labyrinthine states and the transverse component is taken to be the projection of dipoles onto the domain wall normal at each point. At $10 \mathrm{~K}$, the potential features three minima, the leftmost and rightmost ones being associated with the Néel nature of domain walls. The minimum at zero is associated with dipoles residing at the boundaries of domain walls, and whose orientation is along [001] and [001] directions. Increasing temperature leads to a gradual flattening of the minima ultimately resulting in a single minimum potential at $u_{t r}=0$ for $T \geq T_{i n v}$. The gradual lessening of barrier heights is associated with increased thermal fluctuations of dipoles which lead to more corrugated walls, but also enable the reorientation of the meandering stripes. In this regard, the barrier softening of transverse components of domain walls dipoles undermines surface tension effects and enhances walls fluidity. The loss of configurational entropy subtending the parallel reorientation of the labyrinthine stripes (greater mesoscopic order) is offset by the increase of the vibrational entropy of dipoles (greater microscopic disorder). Frozen in the 'disordered' high-symmetry labyrinthine phase, transverse components of the dipoles melt in the 'ordered' low-symmetry parallel stripe state. In this sense, the inverse transition phenomenon, although seemingly counterintuitive, is only inverse from the mesoscopic symmetry standpoint as it can be fathomed without violating the laws of thermodynamics nor relying on a paradoxical inverse entropic scenario ${ }^{3,16,18}$.

This configurational entropy reduction can be rationalized by regarding the labyrinthine domain pattern as a fragmented, mosaic state composed of tiles whose ground-state morphology is a locally parallel stripe arrangement of domains. Such local realization of mesoscopic order within each tile is favored by the dipolar interaction that stabilizes parallel adjacent stripes. Within the mosaic ansatz, denoting by $\xi$ the typical lateral length of a tile and by $L$ the lateral size of the supercell, an estimate of the degeneracy of the labyrinthine phase yields $2^{L^{2} / \xi^{2}}$, since each of the $L^{2} / \xi^{2}$ tiles can locally harbor a parallel stripe alignment along either [100] or [010] directions. These exponentially many labyrinthine states are statistically equivalent while being morphologically incongruent $^{18}$. As expected, $\xi$ is a temperature dependent quantity, as it can be seen in Extended Data Fig. 5b. Approaching $T_{i n v}$ from low temperatures, $\xi$ becomes comparable to the lateral size of the supercell $L$, hence indicating the onset of a global symmetry-breaking and long-range parallel arrangement of stripes. We find that the coarsening of structures is conveyed by the diffusion and relaxation of topological defects localized at the junction of different tiles and reconciling discrepancies in their prevailing local orientations and/or wavelengths ${ }^{25}$. The examination of elementary point topological defects indeed shows that the densities $d_{\mid}$and $d_{|| \mid}$of stripe end-points (or convex disclination of $+1 / 2$ Pontryagin charge ${ }^{27,42}$ ) and three-fold junctions (or concave disclinations of $-1 / 2$ Pontryagin charge ${ }^{27}$ ), respectively shown in Figs. 4b and c, feature a gradual lessening upon approaching $T_{\text {inv }}$ from low temperatures (Fig. 4d). We find that domain coalescence is driven by the recombination/annihilation of defects ${ }^{28,29}$, whereby, for instance, a pair of concave-convex disclinations rebinds into a diffusing dislocation (inset of Fig. 4d) yielding a straightening of the labyrinthine pattern ${ }^{3,30}$.

Rather unexpectedly, we find that these modulated phases (stripe and labyrinthine domain arrangements) are endowed with memory. Upon applying an electric field perpendicular to the film plane, the stripe domains ground state transforms into a nano-bubble phase before yielding a monodomain state at high enough electric field values $^{34}$. We find that the labyrinthine state exhibits an equivalent sequence of electric-field-induced morphological transitions, i.e., from labyrinthine to bubble to monodomain states with increasing magnitude of external field. The two bubble states obtained from either the stripe domains or the labyrinthine ones are energetically equivalent. Notably, upon releasing the stabilizing external field, each of the two bubble states relaxes back to its parent state morphology, obtained priorly to any electric field treatment. This can be seen in Figs. 4e to g that provide the temporal relaxation as obtained from molecular dynamics of each of the bubble states upon removal of the field. This history dependent behavior roots in a 
complex energy landscape and attests of an original intrinsic memory effect, whose seed lies in the arrangement of the bubbles array (Extended Data Fig. 8).

In summary, the present work reports inverse phase sequence in ferroelectric films, whereby a high-symmetry kinetically arrested labyrinthine phase transforms into the less-symmetric parallel stripe domain structure upon increasing temperature. Such an inverse transition involves pattern straightening and coarsening and is predominantly driven by the relaxation and diffusion of point topological defects. We also experimentally show that these nanometric defects encompass up to fifty times larger conductivity when compared to straight domain wall segments and numerically demonstrate that the selfassembled dipolar configurations are endowed with an original memory effect. Such findings allow to devise and propound novel applications of ferroelectric films in logic and storage devices, as well as in memristors ${ }^{31,33,53}$ for neuromorphic computing.

[1] De'bell, K., MacIsaac, A. B. \& Whitehead, J. P. Dipolar effects in magnetic thin films and quasi-two-dimensional systems. Rev. Mod. Phys. 72, 225 (2000).

[2] Seul, M. \& Andelman, D. Domain shapes and patterns: the phenomenology of modulated phases. Science 267, 476 (1995).

[3] Schupper, N. \& Shnerb, N. M. Inverse melting and inverse freezing: A spin model. Phys. Rev. E 72, 046107 (2005).

[4] Tammann, G. Kristallisieren und Schmelzen (Johann Ambrosius Barth, Leipzig, 1903).

[5] Greer, A. L. Too hot to melt Nature 404, 134 (2000).

[6] Portman, O., Vaterlaus, A. \& Pescia, D. An inverse transition of magnetic domain patterns in ultrathin films. $\mathrm{Na}$ ture 422, 701 (2003).

[7] Gu, Z., Pandya, S., Samanta, A., Liu, S., Xiao, G., Meyers, C. J. G., Damodaran, A. R., Barak, H., Dasgupta, A., Saremi, S., Polemi, A., Wu, L., Podpirka, A. A., Will-Cole, A., Hawley, C. J., Davies, P. K., York, R. A., Grinberg, I., Martin, L. W. \& Spanier, J. E. Resonant domain-wall-enhanced tunable microwave ferroelectrics. Nature 560, 622 (2018).

[8] Feig, L., Yudin, P., Stolichnov, I., Sluka, T., Shapovalov, K., Mtebwa, M., Sandu, C. S., Wei, X.-K., Tagantsev, A. K. \& Setter, N. Controlled stripes of ultrafine ferroelectric domains. Nature Communications 5, 4677 (2014).

[9] Peters, J. J. P., Apachitei, G., Beanland, R., Alexe, M. \& Sanchez, A. M. Polarization curling and flux closures in multiferroic tunnel junctions. Nature Communications 7, 13484 (2016).

I. Kornev, I. A., Fu, H. \& Bellaiche, L. Ultrathin films of ferroelectric solid solutions under a residual depolarizing field. Phys. Rev. Lett.93, 196104 (2004).

[10] I. Kornev, I. A., Fu, H. \& Bellaiche, L. Ultrathin films of ferroelectric solid solutions under a residual depolarizing field. Phys. Rev. Lett.93, 196104 (2004).

[11] Lai, B.-K., Ponomareva I., Naumov, I., Kornev, I. A., Fu, H., Bellaiche, L. \& Salamo, G. J. Electric-Field-Induced
Domain evolution in ferroelectric ultrathin films. Phys. Rev. Lett. 96, 137602 (2006).

[12] Streiffer, S. K., Eastman, J. A., Fong, D. D., Thompson, C., Munkholm, A., Ramana Murty, M. V., Auciello, O., Bai, G. R. \& Stephenson, G. B. Observation of nanoscale 180 degrees stripe domains in ferroelectric $\mathrm{PbTiO}_{3}$ thin films. Phys. Rev. Lett. 89, 067601 (2002).

[13] Zhang, Q., Xie, L., Liu, G., Prokhorenko, S., Nahas, Y., Pan, X., Bellaiche, L., Gruverman, A. \& Valanoor, N. Nanoscale bubble domains in ultrathin ferroelectric films. Advanced Materials 29, 170237 (2017).

[14] Solokhin, M. A., Solokhin, A. V. \& Timofeev, V. S. Phase-equilibrium stability criterion in terms of the eigenvalues of the Hessian matrix of the Gibbs potential. Theoretical Foundations of Chemical Engineering 36, 444 (2002).

[15] Ritort, F. \& Sollich, P. Glassy dynamics of kinetically constrained models. Advances in Physics 52, 219 (2010).

[16] Crisanti, A. \& Leuzzi, L. Stable solution of the simplest spin model for inverse freezing. Phys. Rev. Lett. 95, 087201 (2005).

[17] Parisi, G. Spin glasses and fragile glasses: Statics, dynamics, and complexity. PNAS 103, 7948 (2006).

[18] Leuzzi, L. \& Nieuwenhuizen, T. M. Thermodynamics of the glassy state (Taylor and Francis, New York, 2007).

[19] Bouchaud, J. P. Weak ergodicity breaking and aging in disordered systems. J. Phys. I France 2, 1705 (1992).

[20] Booth, I., MacIsaac, A. B., Whitehead, J. P. \& De'Bell, K. Domain Structures in ultrathin magnetic films. Phys. Rev. Lett. 75, 950 (1995).

[21] Prosandeev, S., Lisenkov, S. \& Bellaiche L. Kittel law in $\mathrm{BiFeO}_{3}$ ultrathin films: a first-principles-based study. Phys. Rev. Lett. 105, 147603 (2010).

[22] Prosandeev S., Wang D., Ren W., Iniguez, J. \& Bellaiche, L. Novel nanoscale twinned phases in perovskite oxides. Advanced Functional Materials, 23, 234-240 (2013).

[23] Kornev, I. A., Lisenkov, S., Haumont, R., Dkhil, B. \& Bellaiche, L. Finite-temperature properties of multiferroic $\mathrm{BiFeO}_{3}$. Phys. Rev. Lett. 99, 227602 (2007).

[24] Eliseev, E. A., Morozovska, A. N., Nelson, C. T. \& Kalinin, S. V. Intrinsic structural instabilities of domain walls driven by gradient couplings: meandering anferrodistortive-ferroelectric domain walls in $\mathrm{BiFeO}_{3}$. http://arxiv.org/abs/1810.06668

[25] Pismen, L. M. Patterns and interfaces in dissipative dynamics (Springer, Berlin, 2006).

[26] Lu, L., Nahas, Y., Liu, M., Du, H., Jiang, Z., Ren, S., Wang, D., Jin, L., Prokhorenko, S., Jia C.-L. \& Bellaiche, L. Topological defects with distinct dipole configurations in $\mathrm{PbTiO}_{3}-\mathrm{SrTiO}_{3}$ multilayer films. Phys. Rev. Lett. 120, 177601 (2018).

[27] Nagaosa, N. \& Tokura, Y. Topological properties and dynamics of magnetic skyrmions. Nat. Nanotechnol. 8 899 (2013).

[28] Newell, A. C., Passot, T., Ercolani, N. \& Indik, R. Elementary and composite defects of striped patterns. $J$ Phys. II France 5, 1863 (1995).

[29] Prokhorenko, S., Nahas, Y. \& Bellaiche, L. Fluctuations and topological defects in proper ferroelectric crystals. Phys. Rev. Lett. 118, 147601 (2017).

[30] Harrison, C., Adamson, D. H., Cheng, Z., Sebastian, J. M., Sethuraman, S., Huse, D. A., Register, R. A. \& Chaikin, P. M. Mechanisms of ordering in striped patterns. Science 290, 1558 (2000). 
[31] Boyn, S., Grollier, J., Lecerf, G., Xu, B., Locatelli, N., Fusil, S., Girod, S., Carretero, C., Garcia, K., Xavier, S., Tomas, J., Bellaiche, L., Bibes, M., Barthelemy, A., Saghi, S. \& Garcia, V. Learning through ferroelectric domain dynamics in solid-state synapses. Nature Communications 8, 14736 (2017).

[32] Yang, J. J., Strukov, D. B. \& Stewart, D. R. Memristive devices for computing. Nat. Nanotechnol. 8, 13 (2013).

[33] Chua, L. Memristor-the missing circuit element. IEEE Trans. Circuit Theory 18, 507 (1971).

Acknowledgements The authors thank the DARPA Grant No. HR0011727183-D18AP00010 (TEE Program), the ARO Grant No. W911NF16-1-0227 and the DARPA Grant No. HR0011-15-2-0038 (MATRIX program). Computations were made possible thanks to the use of the Arkansas High Performance Computing Center and the Arkansas Economic Development Commission. B. X. thanks support from Priority Academic Program Development (PAPD) of Jiangsu Higher Education Institutions. S. Prosandeev appreciates support of RMES 3.1649.2017/4.6 and RFBR 18-52-00029_Bel_a. V.G., S.F. and B. D. thank a public grant overseen by the French National Research Agency (ANR) as part of the Investissements dAvenir program (Reference:ANR-10-LABX-0035, LabexNanoSaclay) and the project EXPAND through ANR-17-CE24-0032, as well as through the PIAF project.

Author Contributions Y. N. conceived the study of the inverse transition phenomenon as a part of a research project about labyrinthine structures initiated by L.B. Y. N. and S. Prokhorenko carried out the simulations and analyzed the data. J. F. elaborated the thin films by pulsed laser deposition, carried out the annealing experiments with the help of C. C., and performed the PFM measurements. B. X. and S. Prosandeev performed additional $\mathrm{BiFeO}_{3}$ simulations. S. F. performed the C-AFM experiments. This experimental work was done under the guidance of V. G. B. D. performed XRD measurements. After a first draft written by Y. N., all authors discussed the results and contributed to the final manuscript.

Author Information Reprints and permissions information is available at www.nature.com/reprints. The authors declare no competing interests. Correspondence and requests for materials should be addressed to Yousra Nahas.
Figure 1. Stripes vs. maze at low temperature. a Ground state dipolar configuration (parallel stripes) in the middle layer of an $80 \times 80 \times 5$ unit cells film of $\mathrm{Pb}\left(\mathrm{Zr}_{0.4} \mathrm{Ti}_{0.6}\right) \mathrm{O}_{3}$ as obtained upon slowly decreasing temperature from $650 \mathrm{~K}$ down to $10 \mathrm{~K}$. b Dipolar configuration of the maze or labyrinthine pattern as obtained upon abruptly cooling the system from $650 \mathrm{~K}$ to $10 \mathrm{~K}$. Gray (red) dipoles are oriented along [001] ([001] $)$ pseudo-cubic direction.

Figure 2. Inverse transition simulations. a Temperature dependence of the orientational order parameter $O_{h v}$ upon slowly heating the labyrinthine state of a $80 \times 80 \times 5$ film of $\mathrm{Pb}\left(\mathrm{Zr}_{0.4} \mathrm{Ti}_{0.6}\right) \mathrm{O}_{3}$. Panels $\mathbf{b}$ to $\mathbf{g}$, where gray (red) dipoles are oriented along $[001]([00 \overline{1}])$ pseudo-cubic direction correspond to $10 \mathrm{~K}, 110 \mathrm{~K}, 185 \mathrm{~K}, 260 \mathrm{~K}, 335 \mathrm{~K}$, and $410 \mathrm{~K}$, respectively and show the evolution with temperature of the labyrinthine domain pattern in the film middle layer. The structure factor plots obtained by Fourier transformation of the z-component of the corresponding dipolar field are also provided, where $a q_{x}$ and $a q_{y}$ are the $\mathrm{x}$ and $\mathrm{y}$ components of the dimensionless wave vector that take values within the $-\pi$ to $\pi$ interval ( $a$ is the in-plane lattice constant). White to pink coloring is done according to the value of the structure factor with white (pink) color corresponding to its lowest (highest) value. The color scale is the same for all plots.

Figure 3. Experimental observation and simulations of the inverse transition in $\mathrm{BiFeO}_{3}$ films. Panel a provides $5 \times 5 \mu \mathrm{m}^{2}$ in-plane piezoresponse force microscopy phase images of a 95-nm-thick $\mathrm{BiFeO}_{3}$ film grown on a (110)-oriented orthorhombic $\mathrm{DyScO}_{3}$ substrate, for the as-grown sample, and the same sample after $773 \mathrm{~K}, 1023 \mathrm{~K}$, and $1073 \mathrm{~K}$ annealing. b In-plane piezoresponse force microscopy of 30-nm-thick $\mathrm{BiFeO}_{3}$ film grown on $\mathrm{SrRuO}_{3}(10 \mathrm{~nm}) / \mathrm{DyScO}_{3}(110)$. The scale bar is $2 \mu \mathrm{m}$. Conducting AFM (current mapping) acquired with $\mathrm{V}_{\mathrm{DC}}=1.7 \mathrm{~V}$ applied on $\mathrm{SrRuO}_{3}$ bottom electrode in periodic stripy areas (bottom left) and defected areas with high conduction spots at three fold junctions (top right) and end-point (bottom right) topological defects. Scale bars are $500 \mathrm{~nm}$. Panel $\mathbf{c}$ shows the distributions of the z-component of polarization (red to green indicate negative to positive values) in a middle layer of $\mathrm{BiFeO}_{3}$ film at different temperatures, as obtained from $36 \times 36 \times 10$ supercell effective Hamiltonianbased Monte Carlo simulations under periodic boundary conditions with ideal short-circuit screening and isotropic misfit strain of $-0.16 \%$. The system was abruptly quenched from $2000 \mathrm{~K}$ down to $10 \mathrm{~K}$ and consequently progressively heated up with 40000 relaxation sweeps at each temperature. In simulations, we find that below $T_{i n v}$, the system exhibits mixed 109 and 71 degrees domain walls, while above $T_{i n v}$, only 109 degrees domain walls are observed. 
Figure 4. Energy, topological defects and memory effects. a Free-energy-like potentials of the labyrinthine domain pattern at $10 \mathrm{~K}, 110 \mathrm{~K}$, and $210 \mathrm{~K}$. Curves are obtained through the computation of the logarithm of the probability distribution functions $\rho$ (averaged over the distributions of one hundred labyrinthine realizations) of the transverse to domain walls component of the local modes, $u_{t r}$, within the $80 \times 80 \times 5 \mathrm{~Pb}\left(\mathrm{Zr}_{0.4} \mathrm{Ti}_{0.6}\right) \mathrm{O}_{3}$ supercell. Note that the local mode $u$ is a vector proportional to the electric dipole moment. $\mathbf{b}$ and $\mathbf{c}$ correspond to stripe end-points and three-fold junctions, respectively. d Evolution with temperature of the densities $d$ (per $\mathrm{nm}^{2}$ ) of stripe end-points $d_{\uparrow}$ and three-fold junctions $d_{\|\|}$. Insets show the evolution with increasing temperature (from top to bottom) of labyrinthine stripes morphology within a portion of the middle layer of the simulated $80 \times 80 \times 5 \mathrm{~Pb}\left(\mathrm{Zr}_{0.4} \mathrm{Ti}_{0.6}\right) \mathrm{O}_{3}$ film. Dark area highlights a local straightening process of neighboring stripes upon increasing temperature. e Temporal evolution at $10 \mathrm{~K}$ of the energy per unit cell of a $56 \times 56 \times 5$ supercell of $\mathrm{Pb}\left(\mathrm{Zr}_{0.4} \mathrm{Ti}_{0.6}\right) \mathrm{O}_{3}$ during the relaxation of the two bubble states upon removal of the external electric field. Dark (bright) curve corresponds to the relaxation of the bubble state obtained from electric field treatment of the labyrinthine (parallel stripes) pattern. Panels $\mathbf{f}$ and $\mathbf{g}$ show consecutive snapshots of such temporal evolutions (from top to bottom) just after removal of the field. Snapshots correspond to the mid layer of the supercell, where dark and white regions represent [001] and [001] dipoles orientations. 


\section{Methods}

\section{Computational details}

We mimic $\mathrm{Pb}\left(\mathrm{Zr}_{0.4} \mathrm{Ti}_{0.6}\right) \mathrm{O}_{3}(\mathrm{PZT})$ ferroelectric ultrathin films that are grown along the (001) direction (which is chosen to be the z-axis) and are $\mathrm{Pb}-\mathrm{O}$ terminated at all surfaces/interfaces. The studied films typically have a thickness of $2.0 \mathrm{~nm}$ (i.e., of 5 unit cells), and are subjected to a compressive strain of $-2.65 \%$ in order to ensure that dipoles have a preferential direction along the out-of-plane direction. Such value would approximately account for the mismatch of lattice constants of the cubic phases of strontium titanate (STO) and PZT. They are interposed between (realistic) electrodes that can only screen $80 \%$ of the polarization-induced surface charges, and modeled by various $L \times L \times 5$ supercells that are all periodic along the $[100]$ and [010] directions while finite along the z-axis. Technically, a first-principles-based effective Hamiltonian ${ }^{34}$ is used within Monte-Carlo (MC) simulations to determine the energetics and local electric dipoles in each perovskite five-atom cell of these supercells. The validity of this approach was demonstrated by previous theoretical studies of ultrathin PZT films under compressive strains that (1) yield 180 degrees up and down stripe domains that periodically alternate along [100] (or along [010]) for their ground-state ${ }^{34,35}$, in agreement with experimental observation ${ }^{36}$ (note that 'up' (respectively, 'down') domains refer to domains in which the z-component of the dipole is parallel (respectively, antiparallel) to the z-axis, respectively); (2) predict a linear dependency between the width of these periodic stripes and the square root of the film's thickness ${ }^{37}$, as consistent with recent measurements ${ }^{38}$; and (3) have also led to the prediction of various topological defects such as vortices ${ }^{39}$, dipolar waves ${ }^{40}$, bubbles ${ }^{41}$ and merons (or convex disclinations) $)^{42}$ in ferroelectrics, that have been experimentally confirmed ${ }^{42-44}$. Note that the predicted temperature has to be rescaled by a factor of $\sim 1.6$ with respect to measurements ${ }^{45}$. It is also worthwhile clarifying the role of thickness in the observed inverse transition. The phenomenon is expected to survive as long as the thickness of the film allows from stripe domain arrangement, where the morphological alteration as the thickness of the film increases should mainly affect the width of domains ${ }^{46}$.

\section{Additional insights from the computations}

Panels a to $\mathrm{f}$ of Extended Data Fig. 1 show the evolution of the parallel stripes ground-state upon slowly increasing temperature. In the references ${ }^{47,48}$, authors study the morphology of equilibrium domain patterns depending on the magnitude of gradient terms within the classical Landau-Ginzburg-Devonshire theory, and find that the labyrinthine-like ground state can be stabilized if the gradient energy is sufficiently reduced. Therefore, we can conclude that in our case the effective gradient energy is above the critical value of the gradient which grants parallel stripes ground state upon slowly annealing the system. In Extended Data Fig. 1g, we provide the temperature variation of the scaled structure factor $\tilde{S}\left(a q_{s}, T\right)$ where $\tilde{S}\left(a q_{s}, T\right)$ is taken as the ratio of $S\left(a q_{s}, T\right)$ to $S\left(a q_{s}, 10 \mathrm{~K}\right), a$ is the lattice parameter and $q_{s}$ is the $q$ point corresponding to the wave length of the striped phase modulation. $S(a q, T)$ is computed as the thermodynamic average of the squared norm of the threedimensional discrete Fourier transform of the $z$ component of local dipoles $u_{z}$. Looking into the behavior of $\tilde{S}\left(a q_{s}, T\right)$, it is readily seen that paraelectricity onsets at $T_{c} \sim 380 \mathrm{~K}$. In Extended Data Fig. 2, we show the evolution with temperature of the specific heat $C$ upon (1) heating the parallel stripes ground-state and upon (2) heating the low-temperature labyrinthine kinetically arrested state. While the first curve only exhibits one peak around $T_{c}$, the second curve features two peaks, one at the inverse transition temperature $\left(T_{i n v}\right)$ and one at $T_{c}$. $C$ is extracted from the supercell energy fluctuations $C=\left(1 / k_{B} T^{2}\right)\left(\left\langle E^{2}\right\rangle-\langle E\rangle^{2}\right)$, where $\langle E\rangle$ corresponds to the average over Monte Carlo sweeps of the internal energy $E$ and $\left\langle E^{2}\right\rangle$ to that of its square, and where $k_{B}$ is the Boltzmann constant.

Extended Data Figs. 3 and 4 provide the probability density functions of the cell-by-cell energies calculated for the labyrinthine domain structure at $10 \mathrm{~K}$ for $\mathrm{Pb}\left(\mathrm{Zr}_{0.4} \mathrm{Ti}_{0.6}\right) \mathrm{O}_{3}$ within a $64 \times 64 \times 5$ supercell. Panels a to $\mathrm{d}$ of Extended Data Fig. 3 refer to the on-site energy, first nearest neighbors (1NN) interaction energy, second nearest neighbors (2NN) interaction energy, and dipole-dipole interaction energy, respectively. Panels a to $\mathrm{c}$ of Extended Data Fig. 4 pertain to the third nearest neighbors (3NN) interaction energy, elastic energy, and electrostrictive energy, respectively. The mappings of each contribution to the energy onto the middle layer of the film are also provided in these figures. It is therein seen that while the on-site energy, the second and third nearest-neighbors interaction energy, as well as the electrostrictive energy feature energy gain at the domain walls, the dipole-dipole interaction is the main counterbalancing cost.

In panel a of Extended Data Fig. 5, we show the dependence on temperature of the dipole-dipole energy density upon heating each of the ground-state parallel stripe domain pattern and the kinetically arrested labyrinthine state. It can be seen that prior to the onset of the inverse transition (around $200 \mathrm{~K}$ ), the excess dipolar energy in the labyrinthine state gradually reduces with increasing temperature as a result of the straightening of meandering stripes. Also provided in this figure is the estimate of the evolution with temperature of the dipolar energy density of a fictive labyrinthine state whose serpentine stripe domains are artificially precluded from straightening. The mismatch between the curves associated with each of the fictive and real evolution of labyrinthine state establishes that the labyrinthine domain pattern effec- 
tively reduces its energy upon increasing temperature by adopting a parallel reordering of its stripes. In panel b of Extended Data Fig. 5, we show the growth with temperature of the tile typical lateral length $\xi$. Upon approaching $T_{i n v}$ from low temperatures, $\xi$ becomes comparable to the lateral size of the supercell $L$, hence indicating the onset of a global symmetry-breaking and long-range parallel arrangement of stripes.

We performed additional first-principles-based effective Hamiltonian simulations ${ }^{4-51}$ for $\mathrm{BiFeO}_{3}$ films of different geometries. Specifically, we simulated thick (with respect to the lattice constant) $\mathrm{BFO}$ films where local modes (proportional to dipoles) are centered on the Asites of the perovskite structure. The supercell size was of $36 \times 36 \times 10$ and subjected to compressive strain of $-0.16 \%$ (Extended Data Fig. 6). We have also examined ultrathin BFO films using the film effective Hamiltonian model where local modes (proportional to dipoles) are centered on the B-sites of the perovskite structure. In this second case, the film thickness was taken to be of 5 unit cells (as in the simulations on PZT ultrathin film), and misfit strain was set to $-0.5 \%$. Partial screening electric boundary conditions at film interfaces were used (Extended Data Fig. 7). Both numerical approaches listed above include antiferrodistortive (AFD) degrees of freedom in addition to variables describing inhomogeneous and homogeneous strain as well as local mode vectors. The employed Hamiltonians incorporate, among other terms, the coupling of AFD and ferroelectric degrees of freedom, as well as short range interactions of each of the two order parameters. Both Extended Data Figs. 6 and 7 show that upon heating the deep-quench-obtained low temperature configurations, the domain pattern gradually transforms into parallel stripes domains. Interestingly, the antiferrodistortive (AFD) vectors feature similar behavior with increasing temperature for both investigated $\mathrm{BFO}$ film geometries. These first-principles obtained numerical results, along with their experimental realizations, demonstrate that the inverse transition phenomenon is robust against boundary conditions, film thickness, as well as screening conditions and misfit strain.

We also provide additional details regarding the discovered memory effect. We found that bubbles emerge from either the labyrinth or the parallel stripes states starting from applied external field value of $32 \times 10^{7} \mathrm{~V} / \mathrm{m}$. Beyond the threshold field of $42 \times 10^{7} \mathrm{~V} / \mathrm{m}$, the system forgets its history and does not relax back to the original state. This value is below the field value of $52 \times 10^{7} \mathrm{~V} / \mathrm{m}$ that induces the transition to the monodomain state. Note that typically, theoretical electric fields are about 20 times larger than the experimental ones ${ }^{52}$. Regarding the seed underlying this memory effect, we found that it roots in the arrangement of bubbles. The array of bubbles obtained from the parallel stripes phase shows two additional peaks in its structure factor plot, at the position of the wave vectors that define the periodicity of the par- ent stripe state. Such peaks are absent in the structure factor characterizing the array of bubbles obtained from the labyrinthine state (Extended Data Fig. 8).

\section{Experimental details}

The $\mathrm{BiFeO}_{3}$ thin film was grown by pulsed laser deposition on a (110)-oriented $\mathrm{DyScO}_{3}$ substrate using an excimer laser. First, a 5-nm-thick electrode of $\mathrm{SrRuO}_{3}$ was deposited at $933 \mathrm{~K}$ under 0.2 mbar of oxygen with a laser frequency of $5 \mathrm{~Hz}$. The 95-nm-thick $\mathrm{BiFeO}_{3}$ film was grown at $933 \mathrm{~K}$ under 0.36 mbar of oxygen with a laser frequency of $1 \mathrm{~Hz}$. The bilayer was then cooled down to room temperature under 300 mbar of oxygen. The $\mathrm{X}$-ray diffraction pattern shows the monoclinic (001) orientation of $\mathrm{BiFeO}_{3}$ with Laue fringes attesting the high quality of the epilayer. Piezoresponse force microscopy (PFM) indicates a homogeneous out-of-plane polarization direction towards the $\mathrm{SrRuO}_{3}$ electrode. The inplane PFM contrast shows two alternating variants with $71^{\circ}$ domain walls (Fig.3a). We conducted successive ex situ annealing experiments under oxygen flow on this sample increasing the maximum temperature from $773 \mathrm{~K}$ to $1073 \mathrm{~K}$, ramping at $20 \mathrm{~K} / \mathrm{min}$ from room temperature and keeping the maximum temperature constant for one hour. The cool down process was limited by the inertia of the oven and we estimate the cooling rate to be around $2 \mathrm{~K} / \mathrm{min}$. The resulting PFM domain structure evolution is displayed in Fig. 3a for annealing temperatures of $773 \mathrm{~K}, 1023 \mathrm{~K}$ and $1073 \mathrm{~K}$. No significant change was reported in the maze-like pattern up to $1023 \mathrm{~K}$, while a profound modification to perfectly straight lines is observed after the $1073 \mathrm{~K}$ annealing. Note that the PFM images were taken on random zones of the $5 \times 5 \mathrm{~mm}^{2}$ sample. While the surface topography shows surface desorption in addition to the preserved step and terrace structure (Extended Data Fig. 9), X-ray diffraction does not reveal any structural changes induced by the successive annealing (Extended Data Fig. 10).

We additionally conducted PFM experiments with an atomic force microscope (Nanoscope $\mathrm{V}$ multimode, Bruker) and external SR830 lock-in detectors (Stanford Research) for simultaneous acquisition of in-plane and out-of-plane responses. A DS360 external source (Stanford Research) was used to apply the AC excitation to the $\mathrm{SrRuO}_{3}$ bottom electrode at a frequency of $35 \mathrm{kHz}$ while the conducting Pt coated tip was grounded. The out-of-plane response is homogeneous in accordance with the homogeneous pristine downward polarization all over the $\mathrm{BiFeO}_{3}$ thin film. Current maps were acquired with the same tip connected to a transimpedance amplifier (TUNA, Bruker) with $\mathrm{V}_{\mathrm{DC}}=1.7 \mathrm{~V}$ applied on the $\mathrm{SrRuO}_{3}$ bottom electrode. Data shows enhanced conduction for labyrinthine defects as reported in Fig. 3b.

$\mathrm{X}$-ray diffraction measurements as a function of temperature were performed using a high-resolution 2-axis diffractometer equipped with a rotating anode generator of $18 \mathrm{~kW}$ (Rigaku), with a Bragg-Brentano geometry and 
a $50 \mathrm{~cm}$ focalisation circle allowing an accuracy as high as $0.0002 \mathrm{~A}$ in $2 \Theta$. The (002) out-of-plane pseudo-cubic Bragg peak of $\mathrm{BiFeO}_{3}$ thin film grown on SRO/DSO is measured between $300 \mathrm{~K}$ and $1160 \mathrm{~K}$ (precision better than $1 \mathrm{~K}$ ) and a step of $20 \mathrm{~K}$. Above $1160 \mathrm{~K}$, the film decomposes. From the measured Bragg peak position, the out-of-plane unit cell parameter is extracted and reported in Extended Data Fig. 11 and shows a quasi-linear variation of the film parameter with temperature which indicates that there is no phase transition up to $1160 \mathrm{~K}$.

As visible from Extended Data Fig. 12, we observe the same features as Yang et al. ${ }^{53}$ in the reciprocal space maps measured in our $\mathrm{BiFeO}_{3}$ thin films and the relative intensity of the "superlattice" peaks is increased after annealing. From the in-plane PFM image after annealing (Extended Data Fig. 12b), we estimate the width of the domains (or the periodicity of the domain walls) to be $90 \pm 5 \mathrm{~nm}$. Consistently, the satellites around the (002) BFO film peak (Extended Data Fig. 12d) correspond to a periodicity of $95 \pm 5 \mathrm{~nm}$. We checked that these features disappear when aligning the X-ray beam parallel to the ferroelectric stripes $(\Phi=0$ degree $)$, and doing the same RSMs around (002). 
[34] Lai, B.-K., Ponomareva I., Naumov, I., Kornev, I. A., Fu, H., Bellaiche, L. \& Salamo, G. J. Electric-Field-Induced Domain evolution in ferroelectric ultrathin films. Phys. Rev. Lett. 96, 137602 (2006).

[35] I. Kornev, I. A., Fu, H. \& Bellaiche, L. Ultrathin films of ferroelectric solid solutions under a residual depolarizing field. Phys. Rev. Lett. 93, 196104 (2004).

[36] Streiffer, S. K., Eastman, J. A., Fong, D. D., Thompson, C., Munkholm, A., Ramana Murty, M. V., Auciello, O., Bai, G. R. \& Stephenson, G. B. Observation of nanoscale 180 degrees stripe domains in ferroelectric $\mathrm{PbTiO}_{3}$ thin films. Phys. Rev. Lett. 89, 067601 (2002).

[37] Lai, B.-K., Ponomareva, I., Kornev, I. A., Bellaiche, L. \& Salamo, G. J. Thickness dependency of 180 degree stripe domains in ferroelectric ultrathin films: a first-principles study. Appl. Phys. Lett. 91, 152909 (2007).

[38] Schilling, A., Adams, T. B., Bowman, R. M., Gregg, J. M., Catalan, G. \& Scott, J. F. Scaling of domain periodicity with thickness measured in $\mathrm{BaTiO}_{3}$ single crystal lamellae and comparison with other ferroics. Phys. Rev. $B$ 74, 024115 (2006).

[39] Naumov, I. I., Bellaiche, L. \& Fu, H. Unusual phase transitions in ferroelectric nanodisks and nanorods. Nature 432, 737 (2004).

[40] Sichuga, D., \& Bellaiche, L. Epitaxial $\mathrm{Pb}(\mathrm{Zr}, \mathrm{Ti}) \mathrm{O}_{3} \mathrm{Ul}-$ trathin Films under Open-Circuit Electrical Boundary conditions. Phys. Rev. Lett. 106, 196102 (2011).

[41] Lai, B.K., Ponomareva, I., Naumov, I. I., Kornev I.A., Fu, H., Bellaiche, L. \& Salamo, G.J. Electric-fieldinduced domain evolution in ferroelectric ultrathin films. Phys. Rev. Lett. 96, 137602 (2006).

[42] Lu, L., Nahas, Y., Liu, M., Du, H., Jiang, Z., Ren, S., Wang, D., Jin, L., Prokhorenko, S., Jia C.-L. \& Bellaiche, L. Topological defects with distinct dipole configurations in $\mathrm{PbTiO}_{3}-\mathrm{SrTiO}_{3}$ multilayer films. Phys. Rev. Lett. 120, 177601 (2018).

[43] Zhang, Q., Xie, L., Liu, G., Prokhorenko, S., Nahas, Y., Pan, X., Bellaiche, L., Gruverman, A. \& Valanoor, N. Nanoscale bubble domains in ultrathin ferroelectric films. Advanced Materials 29, 170237 (2017).

[44] Yadav, A. K. et al. Observation of polar vortices in oxide superlattices. Nature 530, 198 (2016).

[45] Bellaiche, L., Garcia, A. 2 \& Vanderbilt, D. FiniteTemperature Properties of $\mathrm{Pb}\left(\mathrm{Zr}_{1-x} \mathrm{Ti}_{x}\right) \mathrm{O}_{3}$ Alloys from First Principles. Phys. Rev. Lett. 84, 5427 (2000).

[46] Kittel, C. Theory of the Structure of Ferromagnetic Domains in Films and Small Particles. Phys. Rev. 70, 965 (1946).

[47] Eliseev, E. A., Fomichov, Y. M., Kalinin, S. V., Vysochanskii, Y. M., Maksymovich, P. \& Morozovska, A. N. Labyrinthine domains in ferroelectric nanoparticles: Manifestation of a gradient-induced morphological transition. Phys. Rev. B 98, 054101 (2018).

[48] Morozovska, A. N., Fomichov, Y. M., Maksymovych, P., Vysochanskii Y. M. \& Eliseev, E. A. Analytical description of domain morphology and phase diagrams of ferroelectric nanoparticles. Acta Materialia 160, 109-120 (2018).

[49] Prosandeev, S., Lisenkov, S. \& Bellaiche L. Kittel law in $\mathrm{BiFeO}_{3}$ ultrathin films: a first-principles-based study. Phys. Rev. Lett. 105, 147603 (2010).
[50] Prosandeev S., Wang D., Ren W., Iniguez, J. \& Bellaiche, L. Novel nanoscale twinned phases in perovskite oxides. Advanced Functional Materials, 23, 234-240 (2013).

[51] Kornev, I. A., Lisenkov, S., Haumont, R., Dkhil, B. \& Bellaiche, L. Finite-temperature properties of multiferroic $\mathrm{BiFeO}_{3}$. Phys. Rev. Lett. 99, 227602 (2007).

[52] Xu, B., Iniguez, J. \& Bellaiche, L. Designing Lead-Free Antiferroelectrics for Energy Storage. Nature Communications 8, 15682 (2017).

[53] Yang, S. Y., Seidel, J., Byrnes, S. J., Shafer, P., Yang, C.-H., Rossell, M. D., Yu, P., Chu, Y.-H., Scott, J. F., Ager III, J. W., Martin, L. W. \& Ramesh, R. Abovebandgap voltages from ferroelectric photovoltaic devices. Nature Nanotech. 5, 143-147 (2010).

Data availability The data that support the findings of this study are available from the corresponding author upon reasonable request.

Extended Data Fig. 1: Temperature evolution of the parallel stripes ground state. Panels a to $f$ show the evolution with temperature of the ground state dipolar configuration (parallel stripes) in the middle layer of an $80 \times 80 \times 5$ film of $\mathrm{Pb}\left(\mathrm{Zr}_{0.4} \mathrm{Ti}_{0.6}\right) \mathrm{O}_{3}$ upon increasing temperature $(10 \mathrm{~K}, 110 \mathrm{~K}, 185 \mathrm{~K}, 260 \mathrm{~K}, 335 \mathrm{~K}$, and $410 \mathrm{~K}$, respectively) where gray (red) dipoles are oriented along [001] ([001] ) pseudo-cubic direction). Panel g provides the temperature variation of the scaled structure factor $\tilde{S}\left(a q_{s}, T\right)$. Vertical dashed line indicates the inflection point of $\tilde{S}\left(a q_{s}, T\right)$ and is taken as the locus of $T_{c}$.

Extended Data Fig. 2: Specific heat of the parallel stripes and labyrinthine states. Specific heat $\mathrm{C}$ as a function of temperature (in arbitrary units). Data is gathered upon slowly heating the ground state parallel stripes domain pattern (1) and the labyrinthine domain pattern (2).

Extended Data Fig. 3: Spatial distribution of on-site, first and second nearest neighbors and dipole-dipole interaction energies. Panelsa, b, c, and $\mathbf{d}$ provide the probability density functions of the cell-by-cell energies (on-site energy, first nearest neighbors $(1 \mathrm{NN})$ interaction energy, second nearest neighbors $(2 \mathrm{NN})$ interaction energy, and dipole-dipole interaction energy, respectively) calculated for the labyrinthine domain structure at $10 \mathrm{~K}$ for $\mathrm{Pb}\left(\mathrm{Zr}_{0.4} \mathrm{Ti}_{0.6}\right) \mathrm{O}_{3}$ within a $64 \times 64 \times 5$ supercell. Each panel provides the contributions stemming from the domains and domain walls, separately. Panels $\mathbf{e}$ to $\mathbf{h}$ provide the mappings of energies onto the middle layer of the film. Blue to red color gradient shows increasing values of unit-cell energies.

Extended Data Fig. 4: Spatial distribution of third nearest neighbors, elastic and electrostrictive energies. Panels $\mathbf{a}, \mathbf{b}$, and $\mathbf{c}$ provide the probability density functions of the cell-by-cell energies (third 
nearest neighbors (3NN) interaction energy, elastic energy, and electrostrictive energy, respectively) calculated for the labyrinthine domain structure at $10 \mathrm{~K}$ for $\mathrm{Pb}\left(\mathrm{Zr}_{0.4} \mathrm{Ti}_{0.6}\right) \mathrm{O}_{3}$ within a $64 \times 64 \times 5$ supercell. Each panel provides the contributions stemming from the domains and domain walls, separately. Panels $\mathbf{d}$ to $\mathbf{f}$ provide the mappings of energies onto the middle layer of the film. Blue to red color gradient shows increasing values of unit-cell energies.

Extended Data Fig. 5: Energetics and spatial correlations at play in the inverse transition. a Evolution with temperature of dipole-dipole energy density upon heating the ground state parallel stripes domain pattern (1) and the labyrinthine domain pattern (2). These two curves meet above $200 \mathrm{~K}$, temperature at which the inverse transition occurs. The third curve (3) corresponds to what would have been the dipole-dipole energy of domain walls if the labyrinthine domain walls would have gradually wiggled with no reordering of the stripes (fictive labyrinthine evolution). b Evolution with temperature of the typical size of locally ordered groundstate tiles composing the labyrinthine domain pattern. Data is obtained via the analysis of structure factors of square patches of varying size at each temperature. Specifically, at each temperature, $\xi$ corresponds to the maximal patch size featuring two-peaked structure factor. Solid line is a guide for the eyes.

Extended Data Fig. 6: Simulations of the inverse transition in thick BFO films. Panels $\mathbf{a}$ and $\mathbf{b}$ illustrate the evolution with temperature of the domain pattern in BFO in terms of the distribution of the ferroelectric and antiferrodistortive (AFD) order parameters, respectively. Results were obtained through MC simulations using the effective Hamiltonian scheme of a $36 \times 36 \times 10$ film subjected to a $-0.16 \%$ misfit strain, with periodic boundary conditions. The system was abruptly quenched from $2000 \mathrm{~K}$ down to $10 \mathrm{~K}$ and consequently progressively heated up with 40000 relaxation sweeps at each temperature. It is seen that the distributions of both ferroelectric and antiferrodistortive order parameters exhibit the inverse transition with $\mathrm{T}_{\text {inv }} \sim 1100 \mathrm{~K}$ and $\mathrm{T}_{c} \sim$ $1300 \mathrm{~K}$ (these numerically predicted temperatures are in good agreement with our experimental findings). We find that below $T_{\text {inv }}$, the system exhibits mixed 109 and 71 degrees domain walls, while above $T_{i n v}$, only 109 degrees domain walls are observed. In panel a, dipoles are colored according to their z-component while in panel $\mathbf{b}$, AFD vectors are colored according to the $\operatorname{atan}\left(\mathrm{W}_{y} / \mathrm{W}_{x}\right)$, where $\mathrm{W}_{y}$ and $\mathrm{W}_{x}$ denote the $\mathrm{y}$ and $\mathrm{x}$ components of the AFD local vectors.

Extended Data Fig. 7: Simulations of the inverse transition in thin BFO films. Panels $\mathbf{a}$ and $\mathbf{b}$ illustrate the evolution with temperature of the domain pattern in BFO in terms of the distribution of the dipolar and AFD order parameters, respectively. Results were obtained through MC simulations using the effective Hamiltonian scheme of a $36 \times 36 \times 5$ film subjected to a $-0.5 \%$ misfit strain with open boundary conditions, a partial screening at film interfaces (effective screening parameter $\beta=0.5$ ). The system was abruptly quenched from $2000 \mathrm{~K}$ down to $10 \mathrm{~K}$ and consequently progressively heated up with 40000 relaxation sweeps at each temperature. It is seen that the distributions of both ferroelectric and antiferrodistortive order parameters exhibit the inverse transition with $\mathrm{T}_{\text {inv }} \sim 525 \mathrm{~K}$ and $\mathrm{T}_{c} \sim$ $650 \mathrm{~K}$. We find that the system exhibits 71 degrees domain walls. In panel a, dipoles are colored according to their z-component while in panel $\mathbf{b}, \mathrm{AFD}$ vectors are colored according to the $\operatorname{atan}\left(\mathrm{W}_{y} / \mathrm{W}_{x}\right)$, where $\mathrm{W}_{y}$ and $\mathrm{W}_{x}$ denote the $\mathrm{y}$ and $\mathrm{x}$ components of the AFD local vectors.

Extended Data Fig. 8: Origin of the memory effect. Panels $\mathbf{a}$ and $\mathbf{b}$ show the structure factor plots for the bubbles configurations $\mathbf{c}$ and $\mathbf{d}$, respectively. Panels a and $\mathbf{c}$ correspond to the bubble state at $10 \mathrm{~K}$, as obtained upon applying a field of $40 \times 10^{7} \mathrm{~V} / \mathrm{m}$ to the parallel stripes configuration, while panels $\mathbf{b}$ and $\mathbf{d}$ correspond to the bubble state at $10 \mathrm{~K}$, as obtained upon applying a field of $40 \times 10^{7} \mathrm{~V} / \mathrm{m}$ to the labyrinthine configuration.

Extended Data Fig. 9: Imaging of the domain structure evolution with temperature in $\mathrm{BiFeO}_{3}$ sample. Topography, in-plane PFM phase and amplitude of (a-c) the as-grown sample, and the same sample after (d-f) $773 \mathrm{~K}$ annealing, (g-i) $1023 \mathrm{~K}$ annealing, and (j-1) $1073 \mathrm{~K}$ annealing. Each annealing step was one hour long. The scale bar is $4 \mathrm{~nm}$ in $\mathbf{a}, \mathbf{d}, \mathbf{g}$ and $10 \mathrm{~nm}$ in $\mathbf{j}$.

Extended Data Fig. 10: XRD characterization of domain patterns in $\mathbf{B i F e O}_{3}$ sample. $2 \Theta-\omega$ X-ray diffraction patterns of the as-grown $\mathrm{BiFeO}_{3}$ sample and the same sample after the successive annealing up to $1073 \mathrm{~K}$. a full scale and b zoom around the (001) peak.

Extended Data Fig. 11: Evolution with temperature of the lattice parameter of the $\mathrm{BiFeO}_{3}$ sample. Evolution of the out-of-plane parameter upon heating the parallel stripes phase of the $\mathrm{BiFeO}_{3}$ sample. Values were obtained by fitting the XRD data and do not reveal any phase transition up to $1160 \mathrm{~K}$.

Extended Data Fig. 12: Reciprocal space mapping of domain structures in $\mathbf{B i F e O}_{3}$ sample. Ferroelectric and elastic domain structures in a $\mathrm{BiFeO}_{3}$ thin film grown on a (110)-oriented $\mathrm{DyScO}_{3}$ substrate before and after annealing. $2 \times 2 \mu \mathrm{m}^{2}$ in-plane PFM phase images of a $\mathrm{BiFeO}_{3}$ thin film for $\mathbf{a}$, an as-grown sample $\mathbf{b}$, a sample after $1073 \mathrm{~K}$ one-hour annealing. Reciprocal space maps around (002) reflections for the same $\mathrm{BiFeO}_{3}$ thin film $\mathbf{c}$, 
the as-grown sample and $\mathbf{d}$, the sample after $1073 \mathrm{~K}$ onehour annealing. The pink arrows indicate the satellite positions left and right to the (002) film peak. The X- ray beam is aligned at $\Phi=90^{\circ}$, i.e. perpendicular to the stripes. The indices of $\mathrm{DyScO}_{3}$ and $\mathrm{BiFeO}_{3}$ are written in the monoclinic cells. 